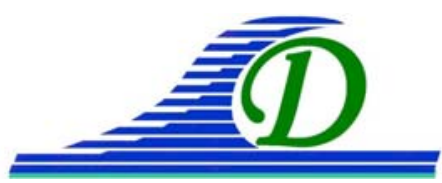

XIII ${ }^{\text {èmes }}$ Journées Nationales Génie Côtier - Génie Civil

Dunkerque, 2-4 juillet 2014

DOI:10.5150/jngcgc.2014.004 @ Editions Paralia CFL

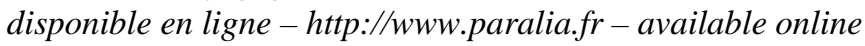

\title{
Dynamique instationnaire des courants d'arrachement de cap
}

\section{Bruno CASTELLE ${ }^{1}$, Giovanni COCO $^{2}$}

\section{CNRS/Université de Bordeaux, UMR 5805 CNRS - EPOC,}

Avenue des Facultés, 33405 Talence Cedex, France.

b.castelle@epoc.u-bordeaux1.fr

2. Universidad de Cantabria, Environmental Hydraulics Institute,

IH Cantabria, Santander, Espagne.

giovanni.coco@unican.es

\section{Résumé :}

Dans ce papier nous modélisons les courants d'arrachement de cap instationnaires sur des bathymétries idéalisées afin de caractériser leur capacité à expulser le matériel flottant (e.g. traceurs passifs, baigneurs) hors de la zone de déferlement. Nos simulations permettent de déterminer, en fonction de la localisation du courant d'arrachement (en amont ou en aval du cap) et de la longueur de la structure par rapport à la zone de déferlement, si celui-ci re-circule ou expulse massivement les traceurs. Lorsque le courant d'arrachement est en amont, i.e. lorsqu'il est induit par la déflection du courant de dérive, il expulse systématiquement les traceurs très loin au large, sauf lorsque l'épi est de dimension si faible qu'il ne perturbe quasiment pas le courant de dérive. Le courant d'arrachement en aval induit par ombrage énergétique des vagues recircule systématiquement et piège les traceurs dans la zone de déferlement, sauf si et seulement si la longueur de l'épi est très largement supérieure à la largeur de la zone de déferlement. Nos résultats suggèrent que les échanges hydro-sédimentaires entre les franges littorales et le plateau sont beaucoup plus importants sur les côtes découpées que le long des littoraux ouverts et que les courants d'arrachement de cap peuvent transporter du sable bien au-delà de la profondeur de fermeture pendant les épisodes de tempête (megarips). Les implications pour la sécurité de la baignade sont également discutées, suggérant qu'il n'existe pas de recommandation universelle quant au comportement à adopter lorsqu'on est emporté dans un courant d'arrachement de cap.

Mots-clés : Courant d’arrachement, Echanges hydro-sédimentaires, Epi, Cap rocheux, Modélisation, Structures lagrangiennes cohérentes, Sécurité de la baignade.

\section{Introduction}

Les courants d'arrachement induits par les vagues sont des courants intenses et étroits dirigés vers le large qui prennent naissance dans la zone de déferlement et s'étendent au large parfois loin au-delà de la zone de déferlement (DALRYMPLE et al., 2011). Ces courants sont très importants pour la sécurité de la baignade; le mélange des masses d'eau et les échanges hydro-sédimentaires entre le plateau continental et les franges 
littorales; le transport des polluants, nutriments, larves; l'érosion localisée de la dune pendant les tempêtes et la morphodynamique court terme (quelques jours à quelques semaines) de la plage. La vitesse des courants d'arrachement fluctue typiquement sur des échelles de temps de $O(1 \mathrm{~min})$ (infragravitaires, associées aux ondes de groupe) et $O(10 \mathrm{~min})$ (fluctuations très basse fréquence). On peut distinguer plusieurs familles de courants d'arrachement (DALRYMPLE et al., 2011). Parmi elles, les courants d'arrachement de cap ont été peu étudiés dans la littérature. Pourtant, ce type de courant est observé quasi-systématiquement le long des caps rocheux, des digues ou des épis d'où leur importance le long des côtes découpées et/ou anthropisées (figure 1).

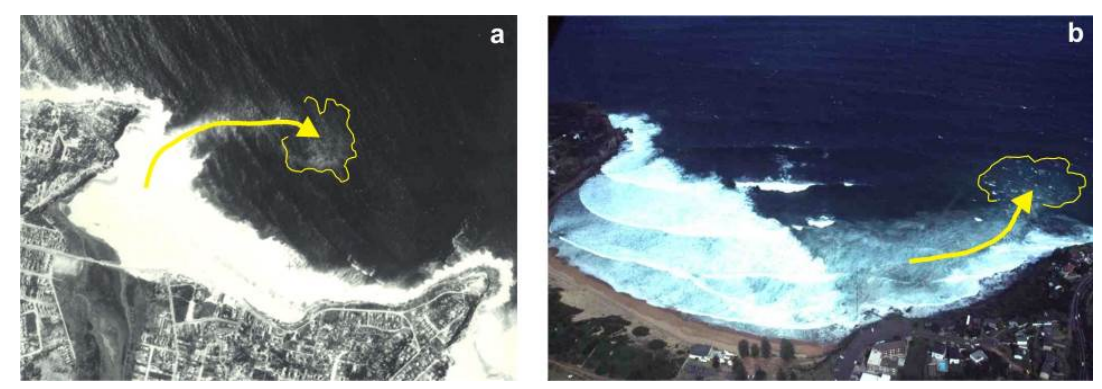

Figure 1. Exemples de courant d'arrachement de cap s'étendant loin au large de la zone de déferlement sur des plages de poche australiennes (Ph. Prof A.D Short).

Les courants d'arrachement de cap peuvent être générés par la déflection du courant de dérive contre l'obstacle en amont ou par ombrage énergétique des vagues en aval (CASTELLE \& COCO, 2012). Par contre, leur capacité à expulser les masses d'eau et les traceurs hors de la zone de déferlement sont très mal compris. Par exemple, certaines étude suggèrent que ces courants d'arrachement re-circulent en permanence (PATTIARATCHI et al., 2009), i.e. n'expulsent aucun matériel en dehors de la zone de déferlement, alors que d'autres suggèrent qu'ils agissent comme un conduit permanent pour transporter le matériel loin au large (SHORT, 2007 ; voir aussi figure 1). Jusqu’à récemment, cette différence extrême de comportement n'était pas expliquée. CASTELLE \& COCO (2013) viennent de montrer par la modélisation numérique que le pourcentage de matériel expulsé par le courant d'arrachement de cap dans les plages de poche peut en effet varier de 0 à $100 \%$ lorsqu'il est respectivement induit par ombrage énergétique ou par la déviation du courant de dérive. En outre, ces comportements peuvent s'inverser lorsque la plage de poche est si étroite que le courant de dérive n’a pas suffisamment de place pour se développer complètement et être ensuite défléchi contre le cap (CASTELLE \& COCO, 2013). Par contre, l'impact de la longueur des caps sur les taux d'expulsion n'a pu être appréhendé. C'est le sujet de cette contribution. 


\section{XIII ${ }^{\text {èmes }}$ Journées Nationales Génie Côtier - Génie Civil \\ Dunkerque, 2-4 juillet 2014}

\section{Méthode}

\subsection{Modèle XBeach}

L'approche de modélisation s'appuie sur le code de recherche 2DH XBeach (ROELVINK et al., 2009) car il permet de simuler la dynamique instationnaire, forcée par les ondes de groupe, dans l'infragravitaire et l'infragravitaire lointain (fluctuations très basse fréquence). Cette dynamique instationnaire est essentielle pour simuler correctement la capacité des courants d'arrachement à expulser le matériel hors de la zone de déferlement (RENIERS et al., 2009). L’interaction houle-courant est également prise en compte et les simulations sont réalisées sans évolution morphologique.

\subsection{Module Lagrangien et taux d'expulsion hors de la zone de déferlement}

Nous couplons le modèle XBeach avec le module Lagrangien développé dans CASTELLE \& COCO (2013) afin de déterminer la capacité des courants d'arrachement de cap à expulser le matériel flottant loin au large. Ce module comprend 2 outils forcés par les vitesses moyennes Lagrangiennes généralisées (i.e., courant total : quasi-eulérien + dérive de Stokes, qui est instationnaire à l'échelle des ondes infragravitaires). Cette approche est nécessaire pour étudier la trajectoire des particules flottantes. Le premier outil permet de suivre des centaines de milliers de particules flottantes passives (drifters). Le second outil calcule les structures cohérentes Lagrangiennes attractives avec un temps d'intégration de 10 minutes, i.e. à l'échelle des fluctuations très basse fréquence (voir CASTELLE \& COCO, 2013). Sans rentrer dans les détails, ces structures sont des barrières de transport "cachées" dans des champs de courant complexes et très instationnaires qui permettent d'identifier facilement les zones de piégeage et les trajectoires préférentielles des particules. Les structures cohérentes Lagrangiennes sont calculées tout au long des simulations, tandis que les drifters sont initialement déposés dans la zone de déferlement à $t=0$ à intervalles réguliers de $2 \mathrm{~m}$. Afin de détecter les drifters sortant de la zone de déferlement, nous utilisons la définition de RENIERS et al. (2009) pour calculer la limite au large de la zone de déferlement.

\subsection{Bathymétries et conditions de vagues}

Afin de s'affranchir des difficultés liées à l'application des modèles aux données réelles (évolution du fond, mauvaise spécification des conditions limite - vagues \& marée, déphasage des ondes de groupe, etc.), nous appliquons le modèle à des cas académiques qui nous permettent d'identifier les conditions de houle et les caractéristiques des caps rocheux impactant les taux d'expulsion. Ici, nous utilisons toujours la même bathymétrie (figure 2) avec une plage barrée homogène d'une longueur de $2 \mathrm{~km}$ s'étendant au large à $600 \mathrm{~m}$ dans $9.5 \mathrm{~m}$ d'eau. Seuls la longueur de l'épi $L$ s'étendant jusqu'au large entre $y=150 \mathrm{~m}$ et $600 \mathrm{~m}$ et l'angle d'incidence des vagues au large $\theta$ 
entre 0 et $20^{\circ}$ sont changés pour chaque simulation. Toutes les simulations sont réalisées sans marée pour des vagues de hauteur significative de $1 \mathrm{~m}$, une période pic de $10 \mathrm{~s}$ et un étalement directionnel de $\mathrm{n}=10$ (étalement en $\cos ^{2 n}$ ).

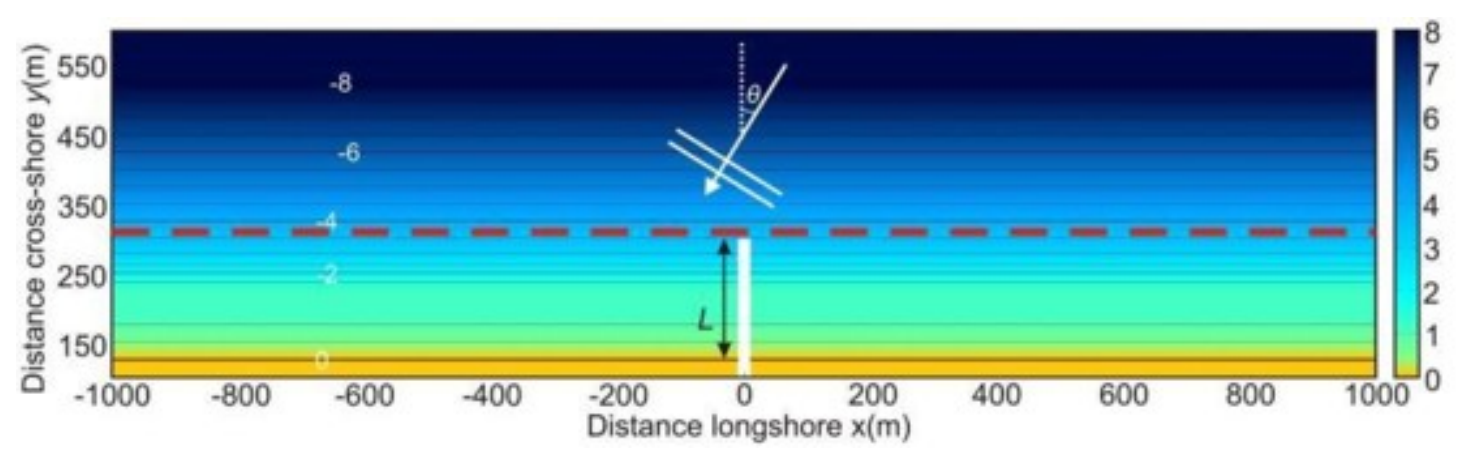

Figure 2. Bathymétrie utilisée pour les simulations avec $L$ la longueur de l'épi et $\theta$ l'angle d'incidence des vagues. La ligne rouge pointillée indique la limite au large de la zone de déferlement.

\section{Résultats}

Nous ne montrons pas ici l'ensemble des simulations mais décrivons 3 simulations représentatives des différents comportements de courant d'arrachement de cap obtenus. Ces 3 simulations sont réalisées pour $\theta=20^{\circ}$, mais avec différentes longueurs de cap $L$ $(60 \mathrm{~m}, 160 \mathrm{~m}, 360 \mathrm{~m})$. La figure 3 montre l'évolution temporelle de la position des drifters et des structures cohérentes Lagrangiennes pour les 30 premières minutes de ces 3 simulations ( $t=0$ après 30 minutes de simulation hydrodynamique sans drifter afin de s'affranchir du régime transitoire).

Pour $L=60 \mathrm{~m}$, i.e. avec un épi ne s'étendant pas au-delà de la zone de surf interne, la trajectoire des drifters est peu perturbée par la structure. Le courant et les drifters contournent l'épi, ces derniers restant dans leur grande majorité à l'intérieur de la zone de déferlement. Une partie des drifters déposés en aval de courant de dérive $(x<0$, en vert) sont piégés dans la petite cellule de circulation associée au courant d'arrachement de cap (en pointillés noirs dans la figure 4a). Le courant de dérive d'intensité de l'ordre de $0.5 \mathrm{~m} / \mathrm{s}$ est peu affecté par l'épi, avec la seule modification notable étant une légère accélération $(\sim 0.6 \mathrm{~m} / \mathrm{s})$ immédiatement en face de la structure en $x=0$ (figure $4 \mathrm{a})$ ). Dans cette situation, le courant d'arrachement de cap peu intense $(\sim 0.2 \mathrm{~m} / \mathrm{s})$ en aval de l'épi est de petites dimensions et n'expulse aucun drifter hors de la zone de déferlement. Le courant d'arrachement de cap en amont est lui aussi de faible intensité et connecte avec le courant de dérive en $y \approx 220 \mathrm{~m}$ et n'expulse qu'un tout petit nombre de drifters hors de la zone de déferlement $(y>300 \mathrm{~m})$. 


\section{XIII ${ }^{\text {èmes }}$ Journées Nationales Génie Côtier - Génie Civil \\ Dunkerque, 2-4 juillet 2014}

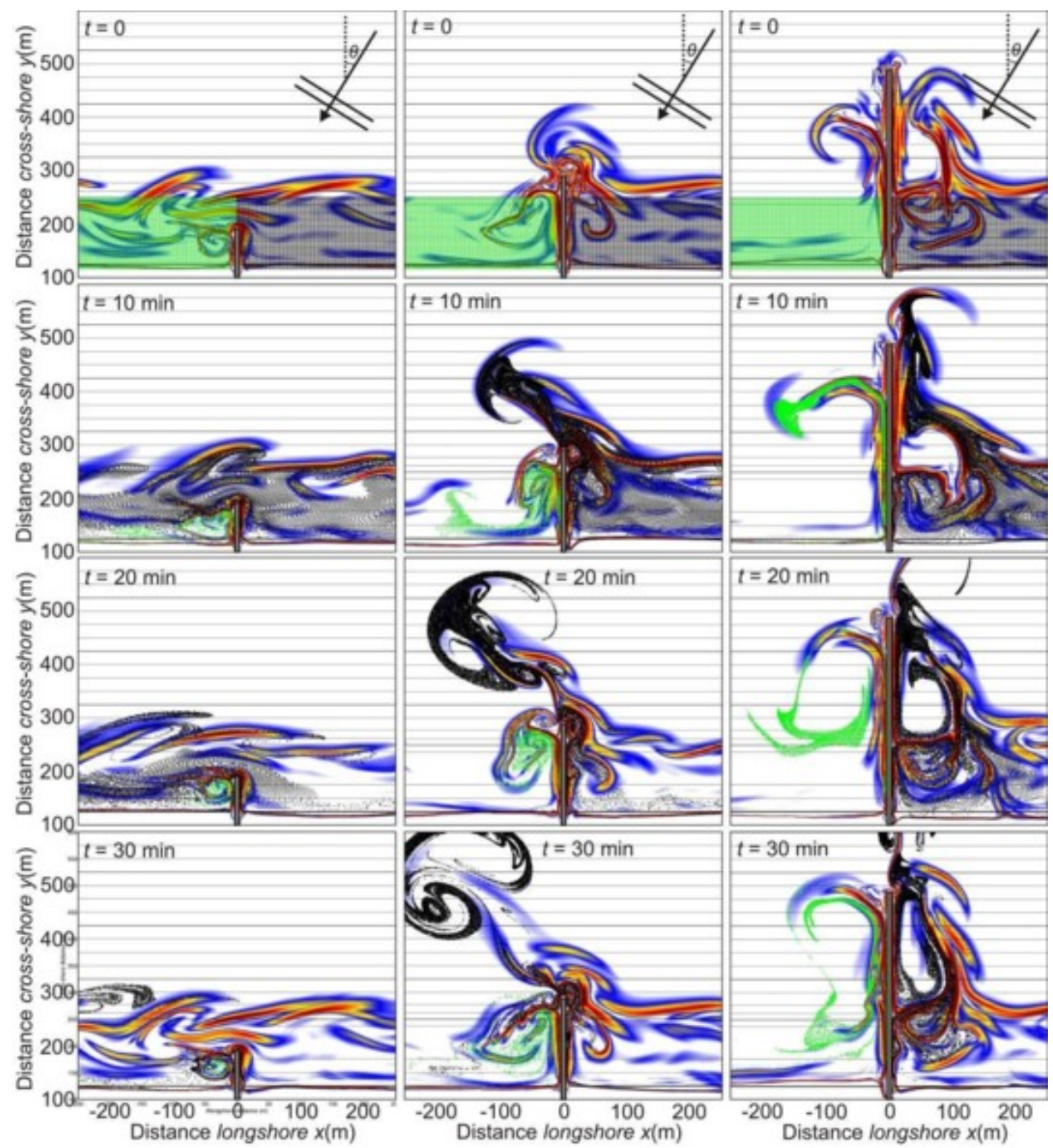

Figure 3. Evolution temporelle des drifters déposés en amont (points noirs) et en aval (points verts), et des structures cohérentes Lagrangiennes (couleurs).

A gauche : $L=60 \mathrm{~m}$; au milieu $L=160 \mathrm{~m}$; à droite $L=360 \mathrm{~m}$.

Dans la simulation avec $L=160 \mathrm{~m}$ (colonne du milieu de la figure 3), le courant d'arrachement de cap en aval de l'épi a des dimensions plus importantes et augmente en intensité ( $0.4 \mathrm{~m} / \mathrm{s}$, pointillés noirs dans la figure $4 \mathrm{~b})$. Comme pour $L=60 \mathrm{~m}$, il recircule en permanence et piège une grande quantité de drifters à l'intérieur de la circulation. Le courant d'arrachement de cap en amont est clairement induit par la déflection du courant de dérive contre la structure (pointillés rouges dans la figure 4b). Cette fois-ci, environ 95\% des drifters pris dans ce courant d'arrachement sont expulsés loin au large (drifter noirs dans la colonne du milieu de la figure 3). Les 5\% restant se 
retrouvent dans la zone de déferlement en aval de l'épi et sont transportés ensuite par le courant de dérive ou sont piégés dans la cellule associé au courant d’arrachement de cap en $x<0$. Pour $L=360 \mathrm{~m}$ (colonne de droite de la figure 4), les 2 courants d'arrachement expulsent massivement les drifters loin au-delà de la zone de déferlement. A notre connaissance, c'est la première fois que ce type de comportement est mis en évidence pour le courant d'arrachement en aval. Ces 2 courants d'arrachement de cap ne sont pas beaucoup plus intenses que ceux obtenus pour $L=160 \mathrm{~m}$ mais s'étendent beaucoup plus loin au large (figure 4c).

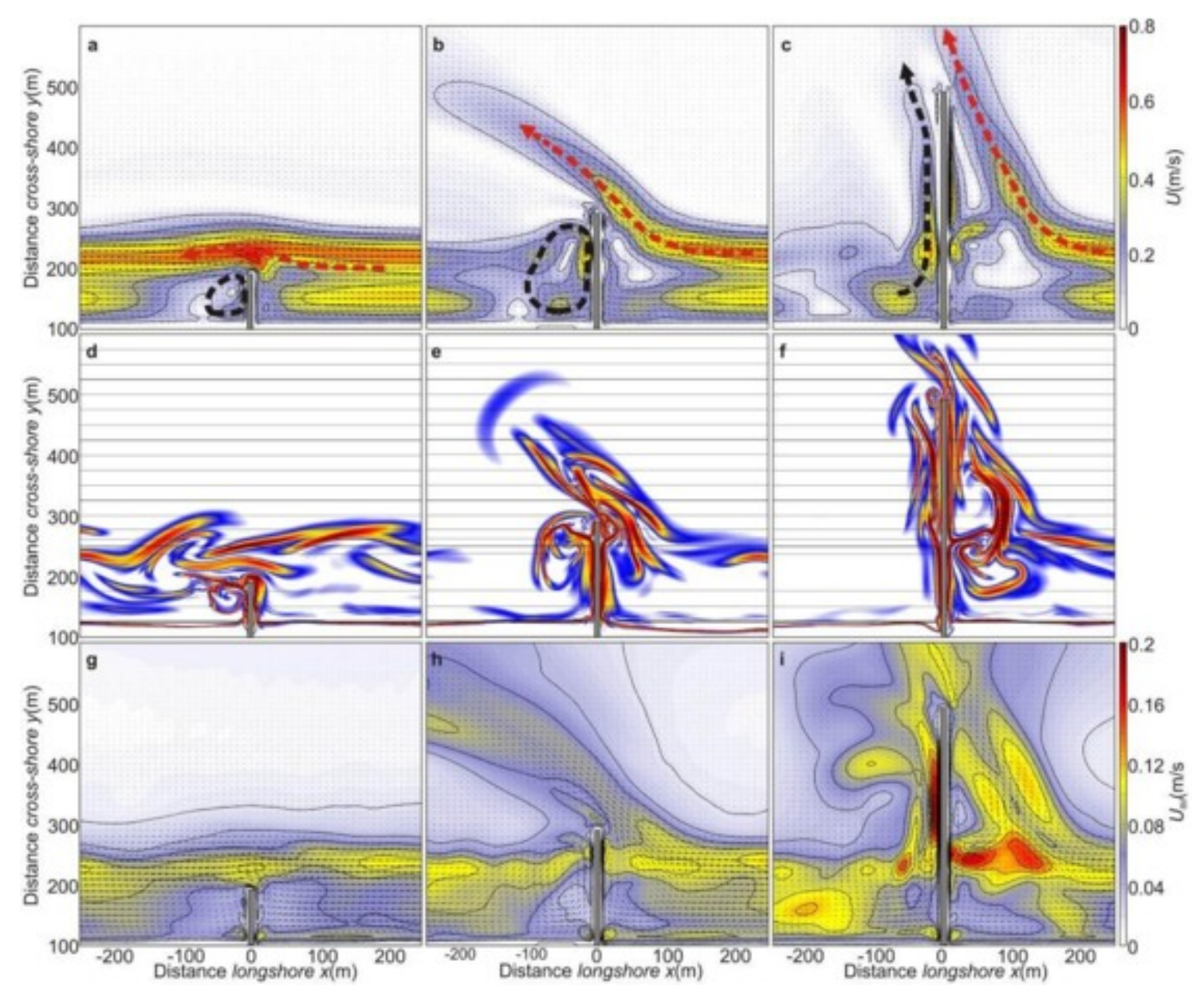

Figure 4. (a,b,c) Circulation moyenne GLM moyennée sur 3 h de simulation; (d,e,f) exemple de structure cohérente Lagrangienne ; $(g, h, i)$ intensité des vitesses moyennes dans la bande de l'infragravitaire lointain (> $10 \mathrm{~min}$ ) calculée sur les vitesses GLM. $L=60 \mathrm{~m}$ (colonne de gauche); $L=160 \mathrm{~m}$ (colonne du milieu); $L=360 \mathrm{~m}$ (colonne de droite).

La figure 4 montre les courants moyens pour ces 3 simulations ainsi qu'un exemple de structure cohérente Lagrangienne et le champ des vitesses très basses fréquence $U_{t b f}$. Clairement, l'expulsion des drifters hors de la zone de déferlement peut être 


\section{XIII ${ }^{\text {èmes }}$ Journées Nationales Génie Côtier - Génie Civil \\ Dunkerque, 2-4 juillet 2014}

diagnostiquée à partir des courants moyens. Les résultats montrent également que la structure des fluctuations très basse fréquence des vitesses devient très complexe dès que le courant de dérive est défléchi contre l'épi ( $L=160 \mathrm{~m}$ et $360 \mathrm{~m}$ ). En particulier ces fluctuations sont observées très loin au large de la zone déferlement. Cette forte intensité des fluctuations très basse fréquence se matérialisent par des vortex instationnaires se décollant du courant d'arrachement qui amplifient le mélange horizontal et la capacité du courant d'arrachement à expulser les drifters loin au large.

\section{Discussion et conclusions}

Ces résultats démontrent que les courants d'arrachement de cap peuvent se comporter très différemment en fonction de la longueur transversale de la structure. Nos simulations permettent de déterminer en fonction de la localisation du courant (amont ou aval) et de la longueur de l'épi par rapport à la zone de déferlement si celui-ci recircule ou expulse massivement les traceurs. Lorsque le courant d'arrachement est en amont, i.e. lorsqu'il est induit par la déflection du courant de dérive, il expulse systématiquement les drifters très loin au large, sauf lorsque l'épi est de dimension si faible qu'il ne perturbe quasiment pas le courant de dérive. Le courant d'arrachement en aval re-circule systématiquement et piège les traceurs dans la zone de déferlement, sauf si et seulement si la longueur de l'épi est très largement supérieure à la largeur de la zone de déferlement.

D’un point de vue de la sécurité de la baignade, il y a actuellement un débat ouvert entre scientifiques et acteurs de la surveillance des plages à l'échelle internationale sur les recommandations à donner lorsqu'un baigneur est pris dans un courant d'arrachement. Les 2 recommandations sont (1) nager parallèlement à la côte et (2) se laisser flotter. Clairement, se laisser flotter est la meilleure solution lorsque le courant d'arrachement de cap est situé en aval de la structure, sauf si celle-ci s'étend loin au-delà de la zone de déferlement. Par contre, pour le courant d'arrachement situé en amont, aucune solution n'est réellement efficace. Les courants d'arrachement de cap ont donc un comportement très différent de celui des courants d'arrachement couramment observés le long des plages ouvertes. (1) Ils peuvent expulser beaucoup plus efficacement les traceurs loin au-delà de la zone de déferlement ce qui suggère que les échanges hydro-sédimentaires entre les franges littorales et le plateau sont beaucoup plus importants sur les côtes découpées que le long des littoraux ouverts. Même si ici nous n’avons pas étudié le transport des sédiments, il est très vraisemblable que les courants d'arrachement de cap puissent transporter de grandes quantités de sable loin au large, bien au-delà de la profondeur de fermeture, pendant les épisodes de tempête (megarips). Ceci pourrait notamment expliquer les budgets sédimentaires négatifs de certaines plages de poche ou des plages ouvertes après la mise en place de structures en dure (e.g. épis, jetées). (2) Alors que les fluctuations très basse fréquence des courants sont très importantes pour l'expulsion des traceurs par les courant d'arrachement le long des plages ouvertes 
(RENIERS et al., 2009), les courants moyens associés aux courants d'arrachement de cap sont suffisants pour caractériser leur capacité à expulser le matériel au large.

Les recherches menées sur les courants d'arrachement de cap doivent être poursuivies, notamment en combinant la modélisation numérique avec les premières mesures Lagrangiennes extensives de courants d'arrachement de cap (MCCARROLL et al., 2014) et le suivi vidéo (e.g. station sur le phare de Biarritz pilotée par IVS, http://sirena.univ-pau.fr/). Ces recherches permettront de valider nos résultats qui sont pour l'instant essentiellement basés sur l'analyse de cas académiques.

\section{Remerciements}

Travail soutenu par le programme national EC2CO-Biohefect/Ecodyn/Dril/ McrobiEN (Dynamique instationnaire et morphodynamique des courants d'arrachement).

\section{Références bibliographiques}

CASTELLE B., COCO G. (2012). The morphodynamics of rip channels on embayed beaches. Continental Shelf Research, Vol. 43, pp 10-23. http://dx.doi.org/10.1016/j.csr.2012.04.010

CASTELLE B., COCO G. (2013). Surf zone flushing on embayed beaches. Geophysical Research Letters, Vol. 40, pp 1-5, http://dx.doi.org/10.1002/grl.50485

DALRYMPLE R.A., MACMAHAN J.H., RENIERS A.J.H.M., NELKO V. (2011). Rip currents. Annual Review of Fluid Mechanics, Vol. 43, pp. 551-581.

MCCARROLL R.J., BRANDER R.W., MACMAHAN J.H., TURNER I.L., RENIERS A.J.H.M., BROWN J.A., BRADSTREET A., SHERKER S. (2014). Evaluation of swimmer-based rip current escape strategies. Natural Hazards, Vol. 71(3), pp 18211846. http://dx.doi.org/10.1007/s11069-013-0979-1

PATTIARATCHI C., OLSON D., HETZEL Y., LOWE R. (2009). Wave-driven circulation patterns in the lee of groynes, Continental Shelf Research, Vol. 29, pp 1961-1974. http://dx.doi.org/10.1016/j.csr.2009.04.011

RENIERS A.J.H.M., MACMAHAN J.H., THORNTON E.B., STANTON T.P., HENRIQUEZ M., BROWN J.W., BROWN J.A., GALLAGHER E. (2009). Surf zone retention on a rip-channeled beach, Journal of Geophysical Research, Vol. 114, http://dx.doi.org/10.1029/2008JC005153

ROELVINK J.A., RENIERS A.J.H.M., VAN DONGEREN A., DE VRIES J.V., MCCALL R., LESCINSKI J. (2009). Modelling storm impacts on beaches, dunes and barrier islands. Coastal Engineering, Vol. 56, pp 1133-1152. http://dx.doi.org/10.1016/j.coastaleng.2009.08.006

SHORT A.D. (2007). Australian rip systems - Friend or foe, Journal of Coastal Research, Special Issue 50, pp 7-11. 